\title{
Three Steps Towards a Theory of Informal Logic
}

\author{
SEALE DOSS Ripon College
}

Formal logic being the kind of enterprise it is, there are many ways one can be led to doubt the legitimacy of such logic. The effort to reduce even a very simple case of ordinary reasoning to a pattern of symbols can lead one to realize, for instance, how dogmatic formal logic really is-just as the attempt to contrive examples of ordinary reasoning which will illustrate such patterns may lead one to sense how misleading it is.

Beyond the barriers of symbolization lie other sources of doubt; for example, the truth-table for conditionals is enough by itself to lead one to suspect that formal logic is wrong, just basically wrong in its effort to capture and clarify the nature of correct reasoning.

It is not surprising that one may be led to wonder, then, about some different way of doing logic, about some different way of characterizing correct reasoning. The trouble, of course, is not merely that there is no alternative system available; it is not even clear what an alternative system would look like. In fact, it is not even clear that the alternative to formal logic must be another system of logic at all.

Even so, the mere politics of the matter suggest the need for at least an alternative theory of logic. For without such a theory, without a theory which portrays correct reasoning in nonformal terms, without a theory which will account for correct reasoning in terms other than abstract patterns, it is most unlikely that the enterprise of formal logic can be swayed, much less dismantled. Mere doubt is hardly enough, in logic no less than in other matters of dogma and tradition.

One might ask, then, what an alternative theory of logic would look like, what an alternative theory of logic might involve. Those are the questions I propose to deal with here.

Let me begin by supposing three things about formal logic itself. First, that what we call formal logic is the outgrowth of a fundamental thesis about the nature of correct reasoning; second, that it is this thesis which generates the rubric of formal logic; third, that it is also this thesis which generates the techniques of formal logic.

The thesis, of course, is that correct reasoning is, indeed, a function of formal patterns, patterns which exist independent not only of whatever it is that one is reasoning about, but also independent of anyone actually engaging in such reasoning. Call it Platonism if you like, but this assumption survives even the transition from syllogostic logic to modern logic.[1]

The rubric which it generates includes such things as the definition of formal validity, the distinction between validity and soundness, the distinction between premises and conclusions, the distinction between deduction and induction, and even the notion that reasoning is, in fact, a matter of "argument construction" - in short, the very vocabulary in terms of which we are now all but forced to talk about logic.

Finally, the techniques of formal logic which flow from this basic assumption (and which reinforce the rubric of formal logic) include such things as the transformation of ordinary sentences into propositions, the use of symbols to represent such propositions, the reliance on inference rules and proof procedures in the construction of arguments, and the testing of arguments by examination of their symbolic structure-in short, a devout determination to replace all concern for subject 
matter with the concern for form

That determination, one might add, reflects the sense in which the thesis, the rubric and the techniques of formal logic are quite deeply rooted in Platonism. But the point of such a sketch is not to trace the origins of formal logic. It is, rather, to provide some scope to the question of what an alternative to formal logic might in volve.

One might now rephrase the question at hand, given such a sketch and given the doubts and difficulties which formal logic is quite clearly prey to. How different would an alternative theory have to be? That is, how different from the theory of formal logic must a theory of informal logic be if there is, in fact, to be an alternative theory of logic?

Rephrased that way, the question reflects still another supposition about formal logic: that behind its fundamental thesis, its rubric, and its techniques - all of which add up to an ongoing system of logic-there is also an implicit theory at work. I take it that the theory of formal logic is simply that correct reasoning is reasoning in accordance with a demonstrably valid inference pattern-for example, a pattern such as $((\mathbf{P} \&(\mathbf{P} \rightarrow \mathbf{Q})) \rightarrow \mathbf{Q})$. I also take it that the system as a whole has emerged, over the past twentyfour centuries, as both a reinforcement of this theory and a vindication of it, as well. One may indeed suspect that the theory of formal logic is wrong, for the kinds of reasons already suggested, but there is, after all, a deeply entrenched tradition at stake here. The quest for an alternative theory-that is, a theory which would account for correct reasoning in terms other than formal patterns - is therefore not likely to appeal to the timid. And that, I think, has a great deal of bearing on the question of how different an alternative theory of logic is really likely to be. [2]

A truly radical alternative to the theory of formal logic might, after all, require the sacrifice of the entire system it has generated, to include not only its vocabulary and its techniques but also, alas, its professional endear- ments. What would one be left to teach? Faced with such a peril, one might naturally wish to alter the question at hand still one more time, asking not how different an alternative theory might be, but rather: How much of the tradition of formal logic can be preserved if the theory behind it is replaced?

Let me illustrate this concern by citing, more or less at random, some of the considerations it provokes. In the search for some alternative to the theory of formal logic, one may wonder just how much of the system it has generated can be left intact by asking, for example, whether or not such rules as modus ponens and modus tollens can be saved. They do, after all, seem quite legitimate. But what shall be their justification, as rules, if one abandons the theory they are drawn from? If the theory of formal logic is abandoned, how shall one be able to talk, even, about such things as antecedents and consequents? About the properties of conditionals? About the reasons why a conclusion can't be drawn from a conditional and the denial of its antecedent? About the reasons why a conclusion can be drawn from a conditional and the denial of its consequent? For that matter, how shall one be able to talk about disjunctions and the law of excluded middle? About conjunctions and the law of contradiction? If one cannot employ symbols, because the theory which justifies their use has been discarded, how can one make sense of the reasons why a statement and the denial of that statement can't be joined together logically? As for statements themselves, without appeal to form - and without appeal to propositions which will demonstrate such form - how can one make sense of the way in which a statement such as "Mary is shopping since she's downtown" is logically equivalent to the statement "If Mary is downtown, then she is shopping"'? And so forth.

Such considerations are quite natural; they reflect the hold which formal logic has upon us. Given that hold, it is also quite natural to wonder how we 
could possibly do without the rest of the system. What would happen to the notion of what an argument is? How could we ever prove that an argument is valid if we abandon formal logic? How could we tell the difference between deduction and induction? How could we deal with universal quantification, with the nature of relations, with such things as logical identity? Must we really discard all the things which formal logic has somehow produced, all its categories, distinctions, and innovations? Must we scrap an entire legacy? Or can it somehow be preserved?

These questions can be seductive, to be sure. In light of such considerations one may well retreat from the search for some alternative to formal logic. One may even propose that informal logic-whatever that may turn out to be-is something to be dealt with in a complementary way. What is required, one may suppose, is not an alternative theory but an adjunct theory, a way of accounting for those instances of reasoning whose correctness (or incorrectness) can't very well be dealt with formally. There is, after all, a certain logic to prudence, too.

The trouble with such a response is not merely that it would be timid. What is worse is that it would evade the issue altogether. What is required, in facing up to the kinds of things which make one suspicious of formal logic, is not some patch-work attempt to save what is useful in it. Nor some politically cautious effort to treat in a complementary (or adjunct) way those matters which lend themselves to informal analysis. What is required, if one is to join the issue head-on, is an outright acknowledgement that the theory of formal logic is quite simply and quite fundamentally wrong. And that, I propose, would be the first step toward a genuinely alternative theory about the nature of correct reasoning.

One fairly simple example will illustrate the sense in which the theory of formal logic is wrong. The example will also indicate what must be done if one is to take the second step toward an alternative theory. It involves what we have come to call "counterfactual conditionals," and so it involves a matter with regard to which formal logic is particularly vulnerable.

Suppose, now, two historians dealing with the American Civil War and its aftermath are led to disagree about what might have happened had the North not won the war. In speculating about such matters one historian is led to claim (as a great many have) that "If the South had won the war, then slavery would have survived in America for another century." That is, of course, a counterfactual claim. Suppose the other historian replies by saying "No, that isn't so. Even if the South had won the war, slavery would have disappeared in another generation." People do, indeed, quarrel about such matters, marshalling the kind of evidence which makes the study of history the subject matter that it is. [3]

From the standpoint of formal logic, such a quarrel would appear to be about the truth of the conterfactual itself, about the truth of a claim which would appear to have the form $(\mathbf{P} \rightarrow \mathbf{Q})$, and which has been made in context within which $P$ (that is, the proposition "The South won the Civil War") is admitted by all to be, in fact, quite false. From a strictly formal standpoint there would seem to be no room at all for such a quarrel, of course. For if $\mathbf{P}$ is false, then $(\mathbf{P} \rightarrow \mathbf{Q})$ must be true, given the formal analysis of conditionals. And so must $(\mathbf{P} \rightarrow-\mathbf{Q})$, for that matter. That is, one might just as well claim that "If the South had won the war, then slavery would not have survived in America for another century" and that, too, would be logically true in terms of formal analysis. For if the truth-table structure for conditionals is correct, then both $(\mathbf{P} \rightarrow \mathbf{Q})$ and $(\mathbf{P} \rightarrow-\mathbf{Q})$ must be true if $P$ itself is false.

That is puzzling enough, no doubt. But there is not just a puzzle involved here; there is also an outright absurdity. For in this example (where $\mathbf{P}$ stands for "The South won the Civil War" and $Q$ stands for "Slavery would have survived in America for another century") 
the second historian, in denying the claim that "If the South had won the Civil War, then slavery would have continued in America for another century", would apparently be asserting that the conditional represented by $(\mathbf{P} \rightarrow \mathbf{Q})$ is false. But the only way in which $(\mathbf{P} \rightarrow \mathbf{Q})$ can be false, according to the truth-table for conditionals, is for $\mathbf{P}$ to be true and $\mathbf{Q}$ to be false. In denying what is represented by $(\mathbf{P} \rightarrow \mathbf{Q})$ the second historian would allegedly be asserting ( $\mathbf{P} \&-\mathbf{Q})$, therefore-and that would certainly include the assertion of $\mathbf{P}$ itself. Must one actually claim, then, that the South did win the Civil War in order to deny the claim that "If the South had won the Civil War, then slavery would have survived in America for another century'? That seems more than puzzling. That is, in fact, quite absurd. And yet that is exactly what the theory of formal logic would force upon one as an instance of "correct reasoning."

Aberrations of this sort are common enough. So are the ad hoc ways by means of which an advocate of formal logic might seek to suppress them. What is needed, one might insist, is a retranslation, a proposition which will fit into a safer form. Or perhaps what is needed is a different way of dealing with compound propositions, a different way of getting at their formal structures. Or perhaps what is needed is a richer way of capturing their form, something which embraces all their different modes and tenses. Or perhaps what is needed is....well, just a bit more tampering with the system. But no amount of tampering seems to prevent such anomalies, and the more refined formal logic becomes the less relevant it becomes, as well. Even those who advocate it do not write, or talk, or think, in conformity with its rigid notion of reasoning. They can't. And no wonder, for the formal patterns which the theory presupposes will not fit the way we actually reason. [4]

Nor can they be made to fit, apparently. An alternative notion of logic might very well tell us why this is so, but to get to such an alternative one must not only admit that the theory of formal logic is wrong. One must also acknowledge that it can't be salvaged, either. And that is what I propose as the second step toward an alternative picture of logic: the admission that no amount of ad hoc tampering can save the theory that reasoning, if it is to be correct, must be reducible to formally valid inference patterns.

It is the next step which will prove difficult. For it is one thing to condemn the theory of formal logic. It is another thing to admit that it can't be salvaged. But it is an altogether different matter to anticipate what might replace it. There are, however, certain clues available, clues which indicate the direction which might be followed in taking this third step toward an alternative theory

One of these clues lies hidden in the manner in which formal logic gives way to symbol manipulation. As we know, it is not uncommon for logicians to construct elaborate systems of deduction with no other goal in mind than the exposition of their formal properties. Systems of this sort may be evaluated in terms of their inventiveness, their compactness, their internal consistency, even their fruitfulness-but hardly in terms of their relevance to anything else, and not at all in terms in their casting light on the way one reasons, or ought to reason, about such things as the American Civil War. That is not their purpose. As studies in pure formal logic, they can be developed without concern for any outside application-and as long as they aren't employed externally, as long as they aren't imposed upon some other subject matter, such symbol-systems can remain immune to issues like the problem of counterfactuals. That much may be obvious enough. What may not be so obvious is the clue which is thus provided about the nature of correct reasoning. The clue is this: a purely formal system of this sort can be an exercise in reasoning, all right-in fact, it will be an exercise in reasoning about the interrelationship of sym- 
bols-but the reasoning which it involves may very well conflict with what would count as reasoning in another subject area. What is logically correct with regard to a subject such as symbols may be logically incorrect with regard to a subject such as the American Civil War, for instance. And what that suggests is a long way, indeed, from the theory of formal logic. For what it suggests is that what shall count as reasoning may, indeed, be dependent on the kind of subject matter one is reasoning about.[5]

In the American Civil War case, for example, the logical issue is not really about the formal properties of a counterfactual, at least not from the standpoint of the subject matter of history. The issue there is not about the logical status of $(\mathbf{P} \rightarrow \mathbf{Q})$ when $\mathbf{P}$ is false. Preoccupied by formal considerations, one may rip from the context which surrounds it an assertion such as "If the South had won the Civil War, then slavery would have survived in America for another century" and may be led to think that is what the issue is about. Thinking that way, one will then be forced to embrace the kind of absurdity cited earlier, or else forced to conclude that historians cannot reasonably speculate about such matters. But neither of these two conclusions would be appropriate. No historian has to claim that the South actually won the Civil War in order to deny the claim in question. Nor is such speculation logically unreasonable. If one listens a bit more closely to the way in which the issue is actually joined, one sees exactly what shall count as reasoning about such matters: the assembling of evidence and counter-evidence, the appeal to analogies and counter-analogies, the building up of a context within which the claim in question can be dealt with meaningfully. The historian who denies this claim will cite, as evidence against it, the way in which the Civil War had already changed the South by 1864 , forcing upon the Southern states a network of industry and transportation, forcing slavery to become not only a moral issue but an economic obstacle as well, altering the very nature of the South so deeply that slavery would have been abolished in another generation even if theSouth had won the war. The historian who had originally made this claim will then appeal to counterevidence, or perhaps to some analogy, pointing out perhaps the way in which the Thirty Years War in Europe led to such exhaustion that the forces of economic and social change were arrested there for at least another century or so. An so forth.

One who listens closely in this way may come to wonder, of course, what the point of such an argument really is. If so, one may come to see that reasoning of this sort reflects nothing less than an effort to make sense of things like war and social change, to make sense of history itself. More important, from the standpoint of logical theory, is the recognition that making sense of history does involve a certain kind of reasoning, and that the reasoning which it involves is quite unlike the way in which one deals with, say, a purely formal system of symbols. Hence the insight that what actually counts as reasoning may depend on the kind of subject matter one is reasoning about.

Similar clues are available from a closer look at other subject areas. Indeed, one will soon be overwhelmed by the difference in what counts as reasoning in, say, microbiology as opposed to celestial navigation.[6] Or the difference in the way one is taught to think about economic growth patterns as opposed to the way one is taught to reason about counterpoint and harmonic chord progression in the study of music. Unless one is looking for such clues, one might overlook, or dismiss as simply irrelevant, the way in which inductive reasoning is employed in elementary number theory as opposed to the way induction is employed in the Millikan oil drop experiment and the determination of the charge of an electron. One might even say these are just different kinds of induction, the one axiomatic, the other experimental. And they are, indeed, quite different-as 
different as the reasoning which occurs in classicial mechanics as opposed to statistical mechanics, as different as the reasoning which lies behind theology as opposed to sociology. [7]

A purist, seeing such diversity as a sign of chaos, if not outright derangement, might insist (in a way reminiscent of Plato) that all these different ways of reasoning simply must be reducible to...well, to something uniform. To a basic set of rules, or laws, or principles, perhaps, but to something universal. But to what? CertainIy not to a formal system, no matter how sophisticated. And not even, one may suspect, to a set of laws such as the law of contradiction. [8] If one takes this diversity seriously, if one looks at the detailed way in which reasoning within different subject areas does involve quite different procedures, one will be forced, I think, to treat these differences not as chaos but, in fact, as genuine clues. Clues, that is, to what a legitimate theory of reasoning must embrace.

That is why I propose that the third step toward an alternative theory of logic would be the acknowledgement and the accommodation of this diversity in reasoning procedures. Having taken this step I shall also propose, at least tentatively, what an alternative theory might be. In place of the theory of formal logic, in place of the theory that correct reasoning is a function of demonstrably valid inference patterns, an alternative theory - that is, a theory of informal logic-would be simply this: correct reasoning is a function of the subject matter about which one is reasoning.

Such a theory is, indeed, quite simple. But it paves the way for a range of new considerations - which is why I think it is, at that, a theory as opposed to an act of resignation. [9] Among these considerations there are three which would now become crucial in the study of logic, all of which have been more or less obscured by the search for strictly formal patterns of inference.

The most pressing consideration,
I think, is a study of the way in which any given subject matter is embedded in a context. A rather bizarre example which illustrates this need was related to me some time ago by, in fact, an advocate of formal logic. A happily married man, he was walking along a street when a lady whom he had never, ever, even seen before suddenly stepped in front of him and demanded, furiously, "Look, are you going to marry me or not?" Apparently what bothered him more than anything else was that he dared not answer her in the one way which formal logic dictates, for he knew very well that a "yes" just would not be correct in such a context. This example speaks for itself as still another indictment of formal logic, but what it also indicates is something far too long neglected in the study of logic-namely, the role which context plays in determining subject matter and in determining what shall count as correct reasoning. What we have come to call "ordinary language analysis" has no doubt made us more aware of this role, but I think we still know very little about it, especially from the standpoint of logical theory. While a formalist goes on lifting sentences out of context, and goes on forcing their transformation into propositions, and goes on searching for "missing premises" which would fill in some preconceived pattern of reasoning, what is really needed, instead, is a more thorough understanding of the role which context plays in reasoning.

The second consideration involves the study of fallacies. If reasoning is a function of subject matter, and except for certain subjects (such as matrix algebra) is not at all a matter of formal patterns, the concern for correctness might now seem to lead to a logical vacuum. How is one to determine whether or not some given instance of reasoning, with regard to some given subject matter, is an instance of correct reasoning? Without appeal to form, what shall count as validity? To fill that vacuum what would be necessary, in the further development of a 
theory of informal logic, would be a far more thorough study of what makes some given instance of reasoning fallacious. Instead of its playing a minor role in logic, what is required is a central role for the study of fallacies, one which keys on the search for fallacies peculiar to given subject areas. This search might very well free us from our preoccupation with what we call "arguments" as the basic expression of logical reasoning; it might lead us to abandon the formally-driven notion that there is a fundamental difference between validity and soundness, even that there is a fundamental difference between deduction and induction. But why not? The only difference that really counts, after all, is the difference between correct reasoning and fallacious reasoning, regardless of the subject matter one is concerned with. [10]

That indicates, in turn, what 1 think would be a third major opening in the study of logic: an altogether different way of getting at the nature of theoretical reasoning, in particular the reasoning which occurs in subject areas such as physics. Since it is the theory of formal logic which has generated the distinction between deduction and induction, and with that such issues as the so-called "problem of verification," it ought not to be surprising that we cannot seem to understand how science is even possible. Nor that, in the philosophy of science, we have been led of late to worry about such things as "the incommensurability of opposing theories," as well as the fear that relativism is the price we must pay for understanding how scientific revolutions come about. What is clearly needed is a fresh start, a way of understanding how theories are actually developed in a given subject area, a start toward the realization that what counts as a theory in physics is not governed by the same kinds of rules and procedures which govern what shall count as a theory in, say, literary criticism or, one might add, in the philosophy of logic, either.

That is only a sketch, of course, of what might follow once it is seen that reasoning is a function of subject matter. These three considerations are but hints at what a theory of informal logic would make pertinent. But they do, at least, suggest what an alternative to the theory of formal logic could involve, and so they provide at least a working answer to the questions with which I began. As for what would count as a viable theory of logic, that has also been suggested. Moreover, if the theory is correct, then there ought to be something quite peculiar with regard to the subject matter of logic itself, something peculiar about the way in which one reasons, or ought to reason, about the very nature of logic. And there is: a reasoned recognition that the search for universal forms is both misleading and futile. Hence, again, a theory of informal logic. And hence, again, the three basic steps which have led toward this theory.

\section{Notes}

[1] F.M. Cornford has argued in Plato's Theory of Knowledge (New York: Humanities Press, 1936, p264) that Plato's notion of dialectic "is not what is known as 'Formal Logic' ". However, it is also arguable that in its emphasis upon universality by way of abstraction the theory of formal logic can at least be linked to the general philosophical outlook called "Platonism." If formal logic does rest on such deeply rooted foundations, then the development of an alternative theory will no doubt require epistemological and ontological considerations well beyond the scope of this paper. Still, first steps first.

[2] I take it that the basic function of a theory of logic is to provide an explanation of what governs and determines the correctness of reasoning. It is in this regard that the theory of formal logic appears to be mistaken. But it would be 
equally mistaken to treat the theory of formal logic as a model of what any given logical theory must be, and to expect of an alternative theory that it, too, must therefore provide (or at least have the power to generate) some kind of system of inference rules and procedures. Timidity is sometimes born of such misguided expectations.

[3] That is not to deny (as I acknowledged in the discussion following this paper) that there are deep methodological disagreements among historians, as exemplified in Michael Foucault's L'Archeologie du Savoir (Paris: Editions Gallimard, 1969). But it is to deny such claims as Ernest Nagel's in "The Logic of Historical Analysis" (Scientific Monthly, 74, 1952) that "there appears to be no good reason for claiming that the general patterns of explanation in historical inquiry, or the logical structure of the conceptual tools employed in it, differs from those encountered in the natural sciences". Even Foucault talks (p.15) of an autochtonous "transformation.... in the field of historical knowledge."

[4] The difficulty of fitting formal logic to counterfactuals is only an illustration of the failure of formalism. One might avoid this particular difficulty the way Quine does in Methods of Logic (New York: Holt, Rinehart and Winston, 3rd ed., 1972, p.21) by limiting formal logic to "conditionals in the indicative mood," with the caveat that "the problem of contrafactuals belongs not to pure logic but to the theory of meaning or possibly the philosophy of science." Fair enough: there is no fit. More generally pertinent in this regard is Quine's "maxim of shallow analysis" in Word and Object (Cambridge: MIT Press, 1960, p.160), viz., "expose no more logical structure than seems useful." He paraphrases that as "where it doesn't itch, don't scratch." Again, fair enough; substitute "fit" for "itch" and the point is made.

[5] Witness, for instance, the different ways in which one would go about arguing for (a) the existence, during the Civil War, of a Confederate plot to assassinate Lincoln, (b) the existence, in mathematics, of a continuous function which is not everywhere differentiable. There is even a difference in the way one would argue for (b) and, in those areas where symbol-systems can be interpretively applied (e.g., physics), the way one would argue for (c) the existence of, say, the neutrino particle. Where such techniques of proof and argumentation differ there is surely a difference in what shall count as correct reasoning, i.e., a difference in the logic actually employed.

[6] As discussed following the presentation of this paper, the basic principle of celestial navigation is set forth in American Practical Navigator (Washington: US Govt. Printing Office, 1958, p. 351): "The various celestial bodies.. might easily be imagined as being equally distant from the earth, all located on the inner surface of a vast hollow sphere. This is the celestial sphere. For most purposes of navigation it can be considered an actuality." Thus, when a marine navigator employs a sextant to calculate a ship's position, the logic behind such calculations involves a principle which (one might say) is known to be false. A formalist, persuaded that "anything follows from a false premise," might well be appalled at that; what it illustrates is only that the logic of celestial navigation is peculiar. So, too, for microbiology, in its own way. 
[7] Consider, with regard to theology versus sociology: (a) What would be achieved by giving a polygraph test to some latter-day Bernadette? Nothing? But why not? (b) What would be achieved by ridiculing the fractional oddity of the claim that the average American family has 2,6 children? Nothing. But why not? Because, in each case, the reasoning which characterizes these different subjects is governed by objectives, procedures, and modes of criticism peculiar to the subject matter itself. What should be cultivated (if we are not to be misled) is critical attention to the logic which is thus internal to any given subject. Is it not the lack of such attention that has led, of late, to the futile (and pathetic) effort to incorporate quantum physics and Eastern mysticism?

[8] A husband arrives home late and is chastised by his wife. An argument ensues, punctuated by the usual accusations: he drinks too much, he never takes the children for a walk, etc. Tired of being criticized, the husband finally interrupts her with a challenge: "Mary, do you really love me?" She pauses and then answers painfully: "I do.... and I don't." Has she violated the law of contradiction? Examples of this sort are by no means trivial; one need only reflect upon the logic behind Freud's theory of neuroses, or behind modern theories of personality in general, to see how such theories invoke a principle of consistency that may not be appropriate in such a domain.

[9] Probes in this direction range across a spectrum of such inspirations, from Stephen Toulmin's The Uses of Argument (London: Cambridge Press, 1958) to Robert
Tragesser's Phenomenology and Logic (Ithaca: Cornell Press, 1977). But neither its originality nor the sentiment behind it is of any real importance in the assessment of such a theory. In the long run, its attractiveness is more likely to turn on (a) the clarification of what is meant by "subject matter," and (b) the development of a taxonomic vision richer than, and more fruitful than, the orthodox academic arrangement of "subjects". And these concerns lead back, in turn, to the epistemological/ontological considerations mentioned in Note 1.

[10] How can we tell when reasoning is fallacious, in any given subject area, if there are no universal, subject-independent rules in terms of which we can assess it? The answer cannot be that any given subject area is simply governed by its own internal logic, for (as suggested in Note 7 with regard to psychoanalytic theory) the reasoning in some subject areas may be inherently fallacious. "But how could you ever know that, if reasoning is a function of subject matter?" Etc. What is at stake here is a version of (and perhaps a key to) the problem of epistemological relativism. The rejection of Platonic formalism need not result in relativism, however, nor in Husserl's notion of "psychologism," nor in nominalism; even the logic of symbol-systems is not arbitrary. What is called for, in the face of such assorted perils, is only a patient, real-world study of the way we actually do succeed (and fail) in our effort to reason about the many different kinds of things we reason about.

Professor Seale Doss, Department of Philosophy, Ripon College, Ripon, WI 54971 